\title{
EL EURO Y SUS REFLEJOS LINGÜÍSTICOS EN ITALIANOY ESPAÑOL (2002-2013): ESTUDIO CONTRASTIVO
}

\author{
Cesáreo Calvo Rigual \\ Universitat de València - IULMA
}

\begin{abstract}
Resumen: En este trabajo se analizan de forma contrastiva los materiales de un corpus de textos paralelos de tipo periodístico recopilados en los años 2002 (año de puesta en circulación del euro) y 2013. Hemos extraído más de 200 unidades léxicas (145 del italiano, 61 del español), la mayoría de ellos compuestos con el prefijoide euro-. Este análisis ha puesto de manifiesto divergencias y convergencias de los dos lenguas en el campo de la formación de palabras. E1 estudio se ha completado con el examen de las palabras compuestas con euroencontradas en las fuentes lexicográficas españolas e italianas.
\end{abstract}

Palabras clave: español, italiano, euro, formación de palabras, lingüística contrastiva

Abstract: This paper analyzes contrastively materials of a parallel corpus of journalistic texts collected in 2002 (year of introduction of the euro) and 2013. We have extracted over 200 lexical units (145 Italian, 61 Spanish), most of them compounds with the prefixoid euro-. This analysis has revealed differences and similarities of the two languages in the area of word formation. The study has been completed with the examination of euro- compounds found in Spanish and Italian lexicographical sources.

Keywords: spanish, italian, euro, word formation, contrastive linguistics

\section{INTRODUCCIÓN}

La lengua es un instrumento eminentemente social, es decir, está intimamente ligado a la realidad que le circunda. Es por ello sensible a los cambios que en esta se producen, y acaban reflejándose en aquella de una manera u otra. Esto afecta fundamentalmente al nivel léxico de la lengua, aquel que más directamente se refiere a dicha realidad y que por ese motivo es el más susceptible de sufrir cambios en la lengua, mucho más que la pronunciación o la morfología. En este artículo nos proponemos observar qué efectos tuvo -y sigue teniendo en nuestros días- la puesta en circulación de una nueva moneda -el euro, la llamada moneda única- en dos lenguas, el italiano y el español. 
En el largo proceso de armonización europea (que empezó en 1951 con la creación de la la CECA) se han ido dando pasos más o menos decisivos. Uno de los más importantes ha sido sin duda el acuerdo adoptado por doce de los estados de la Unión Europea para la implantación de una moneda común, que recibió por consenso el nombre de euro. ${ }^{1} \mathrm{El}$ acuerdo entre dichos países se remonta al 16 de diciembre de 1995, aunque su entrada en vigor no se produjo hasta el 1 de enero de 1999. Las monedas y los billetes pudieron ser utilizados por los ciudadanos europeos el 1 de enero de 2002.

Este evento ha generado no solo un nuevo término (euro), sino también otras palabras y unidades léxicas complejas, además de ciertos problemas que su uso ha comportado al integrarse en sistemas lingüísticos diferentes dotados de reglas morfológicas (de formación del plural, de formación de compuestos, de orden de los componentes en las lexías complejas, etc.) diferentes.

Las primeras documentaciones de la palabra se encuentran, lógicamente, el mismo año en el que se produjo la aprobación del nombre de la nueva moneda única, es decir, en 1995, tanto en español como en italiano. ${ }^{2}$

Nuestra intención es comprobar cómo este hecho, en principio extralingüístico, ha repercutido en la lengua española y en la lengua italiana, concretamente en sus respectivos léxicos. Pretendemos de esta forma observar el comportamiento de cada una de dichas lenguas por separado y también en contraste (pues estamos convencidos de que la confrontación de lenguas es un valioso instrumento que ayuda al entendimiento de los fenómenos lingüísticos presentes en cada una de ellas por comparación con la otra). Estudiaremos este capítulo de historia lingüística, en los campos de la Formación de Palabras y de la Lexicología, mediante el análisis de los datos obtenidos de un corpus periodístico recogido en dos momentos concretos: el año 2002, fecha de inicio de la circulación de las monedas y billetes de euros, y el año 2013. Completaremos el análisis de esos datos examinando varias fuentes lexicográficas en los mismos periodos.

${ }^{1} \mathrm{El}$ euro no fue, sin embargo, la primera moneda común europea, ya que con anterioridad se utilizó, con la misma finalidad, la denominada ecu (o más frecuentemente ECU, puesto que se trataba de una sigla: European Currency Unit). El ecu, la divisa base del Sistema Monetario Europeo, fue introducido en 1979 y se mantuvo en vigor hasta la llegada del euro. La existencia del ecu, que no fue respaldado por la circulación de monedas y billetes entre los ciudadanos, no parece haber dejado rastros significativos en ninguna lengua europea, tampoco en español e italiano.

${ }^{2}$ El GRADIT indica, efectivamente, la fecha de 1995 como primera documentación de la palabra, y como fuente el diario La Stampa. En el diario español ABC encontramos el siguiente ejemplo, en una crónica de la reunión de los jefes de gobierno de la UE celebrada el día anterior en Madrid: "La temprana decisión, adoptada a mediodía del viernes, sobre el nombre de la moneda única, el euro, y el escenario para su puesta en marcha, convirtió casi la cumbre de la Presidencia española en una balsa de aceite." (17/12/1995, p. 29). 
El corpus antes citado se divide en estos cuatro subcorpus:

- Prensa italiana 2002: consta de noticias relacionadas con la implantación del euro recogidas en siete publicaciones periódicas italianas ${ }^{3}$ durante la primera semana del mes de enero de 2002. Consta de un total de 113676 palabras.

- Prensa española 2002: consta del mismo tipo de noticias, recogidas en siete publicaciones periódicas españolas ${ }^{4}$ durante el mismo periodo. 121744 palabras.

- Prensa italiana 2013: con noticias que guardan relación con el euro aparecidas durante 2013 en cinco publicaciones periódicas italianas. ${ }^{5}$ 81521 palabras.

- Prensa española 2013: con noticias que guardan relación con el euro aparecidas durante 2013 en cinco publicaciones periódicas españolas. ${ }^{6}$ 93603 palabras.

A propósito de estos corpus consideramos necesario realizar algunas consideraciones preliminares acerca del lenguaje periodístico en ambas lenguas, puesto que existen algunas diferencias notables. En efecto, es un hecho conocido que el periodista italiano, independientemente de la sección del periódico en la que escriba, tiende a expresarse de forma mucho más llamativa e incluso espectacular que sus colegas españoles. Citamos a este respecto a Manuel Carrera Díaz (1996: 114), quien afirma:

Una primera circunstancia caracterizadora es que, en comparación con sus homólogos españoles, el registro léxico de los periódicos italianos es más amplio; o, si no más amplio cuantitativamente [...], sí al menos más variopinto.

Con palabras similares se expresa Giovanna Mapelli (2007: 358), que cita al autor anterior:

${ }^{3}$ Corriere della Sera, Il Messaggero, Il Resto del Carlino, L'Espresso, La Sicilia, Panorama y La Repubblica. En todos los casos se ha recurrido a las versiones en línea de dichos periódicos. La recogida de estos materiales corrió a cargo de los alumnos del curso de Gramática italiana I del curso 2001-2002 de la licenciatura de Filología Italiana de la Universitat de València. Los materiales de cada publicación fueron sometidos al programa de análisis textual DBT (Data Base Testuale versión 3, realizado por el ILC de la Universidad de Pisa), con la finalidad de obtener los siguientes datos: concordancias, frecuencias lingüísticas, índice de legibilidad.

${ }^{4}$ ABC, El Mundo, La Razón, La Vanguardia, Las Provincias, Levante-EMV y La Clave. La recogida corrió a cargo de los mismos alumnos y los materiales se procesaron con el mismo software (DBT).

${ }^{5}$ Corriere della Sera, Il Messaggero, Il Resto del Carlino, La Repubblica e Il Sole 24 ore. Los materiales han sido tratados con el programa de acceso libre AntConc (versión $3.3 .3 \mathrm{~m}$ para $\mathrm{MacOS})$.

${ }^{6}$ ABC, El Mundo, La Razón, Las Provincias y Expansión. Los materiales han sido tratados con el programa citado en la nota anterior. 
Las discrepancias detectadas radican fundamentalmente en las diferencias que existen entre el periodismo italiano y español, puesto que la prensa italiana posee un mayor nivel de perlocutoriedad, es decir, tiende a ejercer directamente un mayor influjo sobre el lector (Carrera Díaz, 1996) y a exasperar el tono sensacionalista y la brillantez de expresión.

Es muy importante consignar estas divergencias, puesto que nos permitirán explicar en parte las notables diferencias en los resultados obtenidos.

Estos corpus son plenamente comparables, puesto que cumplen los requisitos de homogeneidad temática, temporal y también cuantitativa, al estar formados por un número de palabras similar; por todo ello los consideramos válidos y adecuados -dentro de las limitaciones de cualquier corpus- para hacer observaciones pertinentes y llegar a conclusiones fundadas sobre el objeto de estudio elegido (Hartmann 1980: 39).

La implantación del euro tuvo ciertas consecuencias lingüísticas:

a) La creación de nuevas palabras (compuestas o derivadas) y de nuevas unidades léxicas complejas (que llamaremos lexías complejas en español y unità polirematiche en italiano) para satisfacer la necesidad de denominación de nuevas realidades ligadas a la moneda única.

b) Su inserción en cada sistema lingüístico de acuerdo con sus convenciones gráficas, fonéticas, mofológicas e incluso sintácticas.

Una primera cuestión que debemos plantearnos es la naturaleza del segmento /euro/,puesto que el estudio somero de unos pocos ejemplos como los siguientes, extraídos de nuestro corpus, revela su naturaleza poliédrica: ${ }^{7}$

Secondo la più recente rilevazione della Commissione europea, si considera "totalmente eurocompatibile" il 43 per cento delle medie aziende europee. (L'Espresso)

$\mathrm{E}$ i tanto attesi eurosaldi sono finalmente al via. (Il Messaggero)

«Chi vuol essere eurardario» riproduce online il popolare quiz a premi condotto da Jerry Scotti ma con cifre delle vincite già espresse in euro. (Panorama)

Qual è la sua vera visione dell'euro: è un "eurofilo" convinto o un "euroscettico" prudente? (Corriere della Sera)

Proprio per difendersi da questi pericoli è stato appena messo a punto un nuovo strumento controlla-euro. (Panorama)

Anche in questo caso, al di là dell'effetto-euro, saranno le valutazioni sull'andamento dell'economia, oltre che dei tassi, ad orientare il mercato. (Il Resto del Carlino)

En los dos primeros ejemplos nos encontramos ante compuestos endocéntricos en los que el núcleo se encuentra a la derecha y euro- es un

7 Destacamos en negrita las palabras o expresiones sobre las que queremos llamar la atención. 
prefijoide $^{8}$ que tiene la función de determinante (en el primer caso forma un adjetivo, en el segundo un sustantivo). En el tercer ejemplo euro es una unidad léxica independiente (el sustantivo euro) y hace las veces de base de derivación (formada mediante una particular analogía con el sustantivo miliardario, como se explicará más adelante). En el cuarto caben dos interpretaciones: o bien se trata de un derivado por sufijación al haber añadido un sufijo (sufijoide) a la base nominal euro o bien se trata de un compuesto resultante de la unión de dos elementos neoclásicos. El quinto es un compuesto exocéntrico de carácter adjetivo formado a partir del esquema $\mathrm{V}+\mathrm{N}$, uno de los más productivos en la formación de palabras compuestas tanto en italiano como en español. En el último ejemplo nos encontramos ante un compuesto formado por la yuxtaposición de dos sustantivos, con estructura endocéntrica y determinación a la izquierda, como es natural en italiano (y en el resto de las lenguas románicas).

Por tanto, el segmento /euro/, exclusivamente en el significado de 'moneda única europea', parece poseer las siguientes dos caras:

- Constituyente en la formación de compuestos, uniéndose a palabras.

- Unidad léxica independiente, susceptible de producir palabras mediante prefijación, sufijación y composición nativa.

Entre las gramáticas y obras de referencia consultadas únicamente una (NGRAE I: 769) se refiere a nuestro elemento, que considera no un prefijo o prefijoide sino un sustantivo:

El segmento euro es, además de un elemento compositivo similar a los citados, un sustantivo que designa el nombre oficial de la moneda de la Unión Europea. Como elemento compositivo participa en la formación de palabras que designan entidades relacionadas con Europa o con las instituciones europeas: eurocomisario, eurocomunismo, eurodiputado, eurodivisa, europarlamento, entre otros. Como sustantivo, también puede formar parte de palabras compuestas, como en euroconversor o euromonedero.

Como intentaremos demostrar a continuación, en este uso euro no puede considerarse un sustantivo.

\section{EL PREFIJOIDE EURO-}

La mayor parte de las unidades léxicas obtenidas del procesamiento de nuestro corpus corresponden al esquema [euro- + palabra]. Así pues, se trata de un uso de euro- en la posición que habitualmente ocupan los prefijos. En esa

8 Usaremos este término, presente tanto en la bibliografía italiana como en la española, aunque somos conscientes de la existencia de varios otros, como seudoprefijos, raices sufjias (M. Seco), elementos compositivos, etc. 
posición se encuentran dos grupos de constituyentes cuyas fronteras han sido hasta ahora objeto de un intenso debate. Por ello debemos preguntarnos si se trata de un auténtico prefijo o si pertenece al grupo de los llamados prefijoides, para lo que vamos a exponer a continuación, a partir de dos obras de referencia (Varela-Martín 1999: 4997-4998; Iacobini 2004: 85-86) las características de los prefijoides, que los distinguen tanto de las palabras o lexemas como de los afijos:

1. Los prefijoides no coinciden con ninguna preposición, sea esta española, italiana, latina o griega.

2. Los afijoides constituyen una lista abierta, frente a los afijos, que son una lista cerrada. Nuestro formante, de hecho, se ha incorporado recientemente al acerbo léxico de las dos lenguas.

3. Los prefijoides son generalmente bisílabos o trisílabos, frente a las palabras, que tienen un número variable de sílabas y a los prefijos, que suelen ser monosilábicos.

4. Algunos prefijoides pueden aparecer como primero y también como segundo elemento del compuesto. Euro-, sin embargo, ocupa solo el primer lugar: ni en nuestros corpus ni en las fuentes lexicográficas que citaremos después aparece ningún ejemplo con -euro como sufijoide.

5. Un prefijoide puede combinarse con sufijos para formar nuevas palabras (cosa que no pueden hacer los prefijos). Euro- no se combina nunca en nuestro corpus con afijos (solo en dos casos con otros afijoides). Iacobini considera que cuando se da esta posibilidad, el formante debe ser considerado un lexema, puesto que solo los lexemas pueden recibir un afijo: así pues se confirmaría que euro-, en estos casos, es un afijoide.

6. En cuanto a su función, los prefijoides cumplen la de determinante del núcleo del compuesto (que será por tanto endocéntrico, o 'determinativo' en palabras de Iacobini), y el orden habitual de sus componentes será [determinante + determinado], contrario al de las lenguas romances, pero habitual en griego, lengua de la que provienen muchos de estos formantes y por tanto de los llamados compuesto neoclásicos o cultos. Esta característica se cumple en todos nuestros ejemplos.

En resumidas cuentas, nuestro formante cumple cinco de las características anteriores $(1,2,3,5,6)$; la restante se cumple en realidad solo en algunos prefijoides, por lo que, aun siendo importante, no es central para la categoría. Hay que destacar en particular la número 5, que sirve además para no aceptar la afirmación de la NGRAE antes citada, según la cual se trataría de un sustantivo y no de un prefijoide. 
Nuestro prefijoide (no confundir con el preexistente euro- 'de Europa'; 'de la UE') ${ }^{9}$ tiene otra característica destacada: su carácter polisémico, al menos entre los ejemplos recogidos en nuestro corpus. Podemos señalar los siguientes tres sentidos:

a) 'referente a la moneda única': eurobond, euroconvertitore, eurocent, puede tener incluso un sentido físico: eurosindrome;

b) 'referente a la zona euro': eurozona, euroausterità;

c) 'referente a la implantación de la nueva moneda': eurocampanadas, eurobusiness, euro cassiere, eurofesta.

La mayoría de los ejemplos recogidos se refieren a la última acepción (lógicamente en los subcorpus del año 2002) y a la primera, mientras que los de la segunda son netamente menores (y en su mayoría pertenecen a los subcorpus de 2013). A la primera (y también a la segunda), en cambio, pertenecen la mayoría de los compuestos que hoy encontramos en lo diccionarios, como tendremos ocasión de comprobar más adelante.

En los listados siguientes, divididos por lenguas y por años, se ofrecen los resultados obtenidos: ${ }^{10}$

\section{Corpus 2002 español (36)}

euroabusos, euroasesores, eurobanco, eurobillete, eurocalculadora, eurocampanadas, eurocent, eurocepillo, eurocínico, eurocinismo, eurocirculatorio, euroconsultorio, euroconversor, euroconvertidor, eurodelincuencia, euroempleo, euro-euforia, eurofiesta, eurófilo, eurófobo, euroforia, Eurogroup 50, eurogrupo, eurohistérico, euroinundación, euromanía, euromercado, euromoneda, euromonedero, euromundo, europroducto, europtimista, Eurosistema, eurosuperstar, Eurotower, eurozona

\section{Corpus 2002 italiano (104)}

eurardario,eurine,euro-acquisti, euroadeguarsi, euroansia,euroarrotondamenti, eurobanconota, eurobattesimo, eurobiglietti, euro-bollettini, euroborsellino, euro-business, eurobrindisi, eurocaffe, eurocalcolatore, eurocaos, Eurocapodanno, euro cassiere, eurocelebrazioni, eurocent, euro-choc, eurocittadino, eurocompatibile, euroconfusione, euroconfuso, euroconoscitore, euro-consiglieri, euroconsumatore, eurocontanti, euroconversione, euroconvertitore, eurocrisi, eurocuriosità, euro-day, eurodelirio, euroemicrania, euroentusiasta, euro-esordio, eurofanatici, euro-festa, eurofilo, euroforia, euroforico, eurofratellanza, eurogalateo, eurogentleman, eurogatto, Eurogruppo, euro-guida, eurointenditore, EuroItalia, euro-kit, Eurolandia, eurolira, Eurologo, Euromagazine, Euro-mania, Euromio,

${ }^{9}$ Para el comportamiento de este otro prefijo véase el trabajo de García Palacios (2000-2001).

${ }_{10}$ Muchas de las palabras recogidas aparecen con diferentes formas (por ejemplo, con los componentes juntos o bien separados por un espacio o un guion); en este cuadro aparecerá solo una de las formas; pueden encontrarse también el resto en el vocabulario final. 
euro-moneta, euromonetine, euro-nausea, euronervosismi, euronews, euroofferte, euro-onesti, euro-ottimismo, europanico, euro-pensioni, europessimista, europinocchio d'oro, euro-prezzo, europrincipiante, europroblemi, europrocedure, euroquiz, euro-rapinatori, euroresti, eurosaldi, euroscetticismo, euroscettico, eurosciocchezza, eurosciopero, eurosconti, euroshopping, eurosindrome, Eurosistema, Eurosondaggio, euro-sostenitori, euro-spacciatori, eurospesa, eurospicci, eurospiccioli, eurostress, eurotica, eurotilt, eurotismo, euro-tombola, eurotransazione, euroturisti, Euro Superstar, eurounghie, eurovaticano, eurovittoria, euro-volpe, eurozapping, eurozona

\section{Corpus 2013 español (11)}

eurobanco, eurobonos, eurodesencanto, euroejemplo, euroganador, Eurogrupo, euromanía, Eurosistema, (índice) Eurostoxx, (índice) Eurostoxx 50, eurozona

\section{Corpus 2013 italiano (13)}

euro stoxx, euro-area, euro-austerità, euro-identità, euro-sud, eurobond, eurocrisi, eurodelirio, Eurogruppo, Eurolandia, eurosistema, Eurotower, eurozona

El primer hecho que llama la atención es la diferencia numérica entre los dos listados de 2002, puesto que el listado italiano es tres veces más amplio que el del español. No podemos, sin embargo, quedarnos en el puro dato cuantitativo, ni tampoco dejar de buscar una explicación a esta diferencia. A pesar de que el estímulo y la recogida de datos fueron los mismos para ambas lenguas, los compuestos con euro- son mucho más numerosos en italiano que en español. Para explicar esta diferencia hay que recurrir a factores internos y externos. Entre estos últimos ocupa sin duda un lugar preminente el estilo -ya comentadomanifiestamente enfático y grandilocuente del periodismo italiano, que busca el impacto de la noticia a través de un lenguaje rico y llamativo, mucho más que el español, por el contrario algo más pacato -podríamos decir- que el de su homólogo italiano. Eso no quiere decir que entre los periodistas españoles no se encuentren neoformaciones inusuales con el formante euro- ${ }^{11}$ como veremos, pero es indudable que la mayor libertad de uso del lenguaje a la que están habituados los periodistas italianos hace que se lleven la palma en este y en otros terrenos. De hecho, vemos una gran diferencia entre los corpus de 2002 y de 2013: una vez pasada la 'fiebre' del euro y con las aguas ya de nuevo en sus cauces habituales, la creatividad de los periodistas italianos parece haberse calmado y por ello el número de palabras registradas en la prensa de ambos países en la actualidad es similar.

Pero este factor, relacionado con el lenguaje periodístico no explicaría completamente la gran disparidad constatada. Tenemos que recurrir también a

${ }^{11}$ Bastará nombrar, entre los periodístas españoles cuya pluma juega a menudo con el lenguaje, al desaparecido Francisco Umbral. 
factores intralingüísticos, sin duda más interesantes; para ello cabe preguntarse si existen diferencias, tanto cualitativas como cuantitativas, en el campo de la formación de palabras en español y en italiano, y más concretamente en la formación de palabras con prefijoides, que es el uso más habitual de euro-.

Los compuestos con el prefijoide euro- no constituyen una novedad desde el punto de vista categorial, puesto que vienen a engrosar la larga nómina de los compuestos por un prefijoide y un elemento libre, frecuentes no solo en los lenguajes especializados (en los que predomina el tipo elemento ligado + elemento ligado (p. ej. cardiopatía) sino también en la lengua común. Favorece la extensión en el uso de estos compuestos la fácil interpretación que el hablante hace de ellos. De hecho se ha constatado que en italiano la mayor parte de los neologismos formados mediante algún mecanismo de la formación de palabras son palabras compuestas. Así lo indica el reciente estudio de M. Silvia Rati (2012), centrado en los diccionarios italianos, según el cual el 58\% de esos neologismos son compuestos de ese tipo. ${ }^{12}$

A continuación analizaremos varios aspectos que quizá nos hagan ver y nos expliquen las diferencias constatadas.

a) Grafía: aunque pueda parecer un aspecto secundario, resulta pertinente su estudio, en dos aspectos: las diferentes formas utilizadas en su conjunto para representarlos y las variantes de algunos de ellos en particular.

Antes de continuar, sin embargo, debemos advertir de la situación divergente que se da en las dos lenguas, puesto que para el español disponemos de las normas de la Real Academia Española, mientras que en el caso del italiano no las hay, al no existir tal entidad normativa, sin que de ello se derive ni mucho menos un caos ortográfico. Hasta la publicación de la nueva Ortografía (2010) no existían normas explícitas sobre la escritura de las voces prefijadas en español, si bien lo habitual era escribir sus dos componentes juntos, sin guión de separación. A partir de dicha obra se prescribe que se habrán de escribir siempre juntos, con muy pocas excepciones (cuando el segundo elemento es pluriverbal y cuando este comienza por mayúscula). En cuanto al italiano, Elisa Tonani (Enc. It.: s.v. trattino) resume el uso del guion con prefijados a partir de lo dicho por dos autorizados lingüistas -Luca Serianni y Bice Mortara Garavelli-:

(e) indica l'unione di un prefisso o un prefissoide a una parola, di preferenza nei composti occasionali (vetero-forense) e, in genere, nella prima fase della diffusione di un nuovo composto (eco-incentivi), di norma sparisce nei composti stabili, anche se si danno casi opposti.

Entre los compuestos españoles recogidos en 2002 predomina la forma fusionada (26), aunque en 4 casos alterna con la misma forma entrecomillada

12 Lo que no hace sino confirmar las observaciones hechas por Iacobini y Thornton (1992: 48). No nos constan estudios similares para el español. 
(euromoneda, euromonedero, eurosistema, eurozona), existiendo además 2 casos en los que alternan la forma con mayúscula y con minúscula (eurobanco/Eurobanco, eurosistema/Eurosistema). Hay 6 compuestos que aparecen siempre entre comillas: euroasesor, eurocent, eurocepillo, euroempleos, euromercado, europroducto. Las formas entrecomilladas y la oscilación mayúsculas/minúsculas denotan sin duda la inestabilidad y el escaso arraigo de ciertos compuestos. Solo se usa el guión en un caso, seguramente para subrayar una combinación cacofónica: euro-euforia. En suma, parecen seguirse los criterios tradicionales. Es similar la situación en el subcorpus (más reducido) de 2013, donde no hay ejemplos de uso del guión, mientras que en tres casos se alterna la mayúscula con la minúscula inicial (eurobando, euromanía, eurozona). En un caso aparecen tres formas: Eurosistema/eurosistema/"eurosistema".

La situación es mucho más variada en italiano, como consecuencia, seguramente, de la falta de unas directrices claras:

- Sin guión ni comillas: 51 casos, entre ellos varios anómalos gráficamente, pues el segundo componente está todo él en mayúsculas, según un uso periodístico (euroGALATEO, euroSCIOCCHEZZA, euroSTRESS, euroZAPPING), ajeno al español.

- Con los componentes separados por guión: 31 casos. Por ej.: euro-kit, euro-identità, euro-festa, etc.

- Entre comillas: 10 casos. P. ej.: "euroansia", "eurocuriosità".

- Con los dos componentes separados gráficamente: 2 casos: euro cassiere, euro stoxx.

- Alternancia de la forma con guión y sin guión: 8 casos. P. ej.: euroscettico / euro-scettico, eurosciopero/ euro-sciopero, euroshopping/euro-shopping, etc.

- Alternancia de la forma unida y la forma entre comillas: 3 casos: eurocon sumatore/"euroconsumatore", eurodelirio/"eurodelirio", euroforia / "euroforia", etc.

- Otras combinaciones: euroentusiasta/euro-entusiasti / "euroentusiasti", euro-esordio/"uro-esordio", 'Euro-mania', "euroneww" / euroNEWS, "europinocchio d'oro" / Europinocchio d'oro/Euro-Pinocchio d'oro, eurosaldi /'eurosaldi', eurobond / Eurobond / euro bond, eurozona / Eurozona / eurozona.

Las creaciones basadas en juegos de palabras -que cumplen una función eminentemente lúdica- son netamente más frecuentes en italiano, que parece gozar de una mayor flexibilidad en este terreno. De hecho, en español encontramos solo un ejemplo (que aparece también en italiano y que no es, por tanto, demasiado original): 
La euroforia de estos días no puede borrar la reciente imagen de Schröder, Blair y Jospin negociando el grado de subordinación de Europa a Washington al margen de los restantes líderes europeos. (El Mundo).

En italiano encontramos varios casos más:

Ma oggi, e almeno per 6 mesi, ci sarà questo eurotismo e il dio Eurìalo, quello delle elargizioni, si agiterà nella tomba, e quando ognuno di noi avrà finito l'analisi delle eurine, cioè dei propri valori economici... useremo le carte di credito. (Corriere della Sera) ${ }^{13}$

Al supermercato una giovane ha detto però di trovare la nuova moneta sensuale, "eurotica". Una bella invenzione. (Corriere della Sera)

A Madrid, alla Puerta del Sol, è andato in scena "Euroforia": la Spagna ha dato addio alla peseta nel giorno in cui assume, per i prossimi sei mesi, la presidenza dell'Ue e dell'Eurogruppo. (La Sicilia)

Gli euroforici e i bancomatti (Corriere della Sera) ${ }^{14}$

$\mathrm{E}$ affilano le eurounghie per l'estate. (La Repubblica)

c) Mayor variedad de esquemas sintácticos en los compuestos italianos: esta cuestión está relacionada con la de los diferentes significados que este prefijoide adopta, siendo a veces incluso difícil determinar su valor exacto, al ser posible una doble interpretación como euro ${ }^{1}$ 'de Europa' y también como euro- ${ }^{2}$ 'del euro'. Por otra parte, como veremos más adelante, una interpretación simple de la estructura en términos de [sustantivo determinado + preposición (de/di) + euro] no siempre es factible, pues la creatividad periodística (en particular en italiano) estira como un chicle el significado del prefijoide y su relación con el elemento al que acompaña. Aun así, la mayor parte de las palabras compuestas del español tienen esta interpretación sencilla, como por ejemplo en euromanía 'manía del euro', eurofiesta 'fiesta del euro', etc. Muchos casos del italiano se pueden parafrasear también así: eurobattesimo 'battesimo dell'euro', europroblemi 'problemi dell'euro'.

Pero hay muchas otras preposiciones y locuciones preposicionales que pueden ayudar a explicar la relación entre los elementos constituyentes de estas palabras:

- en/in (varias relaciones semánticas): esp. (solo dos casos): eurobonos 'bonos en euros', eurocepillo 'cepillo [de iglesia] en euros'; ital. (al menos 14 ejemplos): eurotransazione 'transacción en euros', eurocaffe 'café [pagado] en euros', etc.

- por/per (causa): esp (dos casos): euro-euforia 'euforia por el euro',

13 Parece claro el juego de palabras entre dos campos, la medicina y la economía, en el caso de eurine, mediante dos palabras similares (eurine/urine) que ponen en paralelo el estado de salud y el estado de la economía.

14 Doble juego de palabras, en un título de un artículo del conocido articulista Beppe Severgnini. 
eurohistérico 'histérico por el euro'; ital. (16 casos): euronausea 'hartazgo por el euro', eurosciopero 'huelga por causa del euro', etc.

- para/per (finalidad, destinatario): esp.: eurocalculadora 'calculadora para euros', euroejemplo 'ejemplo para la zona euro'; ital.: eurocalcolatore.

- sobre/su: esp.: euroconsultorio 'consultorio sobre el euro'; ital. (8 casos): eurocuriosità 'curiosidades sobre el euro', euroquiz 'consurso sobre el euro', etc.

- con/con: esp.: euroabusos 'abusos con el euro'; ital.: eurocompatibile 'compatible con el euro', euroshopping 'compras en euros', etc.

- en relación con: esp. (cinco casos): eurocínico 'cínico en relación con el euro', eurodesencanto 'desencanto en relación con el euro', etc.

- etc.

Hay además un grupo de compuestos que no tienen una interpretación tan transparente como los anteriores, pues precisan de una paráfrasis más larga y compleja para explicar la relación entre el prefijoide y su núcleo. Damos a continuación algunos ejemplos, mucho más númerosos -de nuevo- en italiano:

Las eurocampanadas. La divisa única estará muy presente en las retransmisiones televisivas de las campanadas de Fin de Año. (La Vanguardia) ${ }^{15}$

Bienvenidos al 'euromundo'. Algunos niños vienen al mundo con un pan bajo el brazo, otros, como el recién nacido Mariano Senabre Conde de Sousa, prefieren alardear de su primera paga paterna en euros. $\left(\right.$ El Mundo) ${ }^{16}$

El Corte Inglés vende «europroductos», como calculadoras, bolígrafos detectores de billetes falsos, cajas registradoras, monederos o bandejas clasificadoras de euros. $(A B C)$

En última instancia, solamente un básico rediseño de las políticas y las instituciones de la eurozona haría posible crear una unión de verdaderos "euroganadores". (El Pais) $)^{17}$

Euro cassiere in prima linea. Cambiare 15 mila bilance, mandare a scuola 20 mila dipendenti, sostituire perfino i portagettoni dei carrelli. (Panorama) ${ }^{18}$

Nel corso della conferenza stampa Codacons, Adusbef, Federconsumatori e Adoc "consegneranno l'ambito 'europinocchio d'oro e un consolatorio secondo premio (euro-gatto ed euro-volpe d'argento) verrà assegnato a chi con furbizia e abili stratagemmi ha saputo gabbare i consumatori. (Il Resto del Carlino $)^{19}$

A metà pomeriggio, nelle tasche francesi, gli euro sonanti (o fruscianti) ammontavano già a oltre duecento milioni. Incluse le alcune migliaia afferrate

${ }^{15}$ Las del paso de 2001 al 2002, año de implantación del euro.

${ }^{16}$ Es decir, al mundo en el que existe una nueva criatura, el euro.

17 Países que han ganado con su incorporación a la zona euro.

18 Cajeras que tienen que afrontar los cambios provocados por la introducción del euro en sus puestos de trabajo.

19 Evidente juego de palabras en los que se utiliza el conocido episodio de las aventuras de Pinocho, cuando el zorro (volpe) y el gato se aprovechan de la inocencia de Pinocho para robarle sus monedas. 
al volo dalle casse di un piccolo supermercato della regione parigina assaltato dai primi euro-rapinatori in Francia. (Corriere della Sera) ${ }^{20}$

Ad Acilia presi i primi due "euro-spacciatori". Due uomini bloccati mentre smerciavano, concedendo un piccolo sconto, eroina pagata con le nuove banconote. (Il Messaggero) ${ }^{21}$

L'assalto degli "euroturisti" diventa l'incubo delle cassiere. Spendere le prime monetine per molti è stato il gioco di Capodanno. $\mathrm{E}$ così per pagare un caffè ci vogliono anche dieci minuti. (La Repubblica ${ }^{22}$

d) Combinación con palabras extranjeras, muy superior en el caso italiano:23

- esp.: erocent, Eurogroup, Eurotower, Eurostoxx. En realidad se trata en todos los casos de formaciones que llegan ya así al español, es decir, no se trata del formante español euro- que se une a una palabra extranjera.

- ital.: eurobusiness, eurocent, euro-choc, euro-day, eurogentleman, euronews, euroquiz, euroshopping, eurostress, eurotilt, eurozapping, euro stoxx, Eurotower. Algunos compuestos son del mismo tipo de los del español, pero la mayoría no.

e) Categorías gramaticales de los compuestos con el prefijoide: casi todas estas nuevas palabras se adscriben al grupo de los sustantivos, aunque hay unos pocos casos de adjetivos, como este (único) del español:

el primer día eurocirculatorio. ( La Razón $)^{24}$

En italiano encontramos solo dos adjetivos: eurocompatibile, euroconfuso. Del todo excepcional es la creación de un verbo (euroadeguarsi 'adaptarse al euro') en italiano, fruto en realidad de un juego de palabras basado en la imitación de una expresión proverbial de origen televisivo, como se explica en el ejemplo:

Comunque, se il presidente Ciampi dice che l'euro va bene, se Prodi mette la mano sul fuoco, anche noi ci inchiniamo. Ma la gioia, no. Al massimo, parafrasando una famosa battuta, possiamo dire: non capisco, ma mi euroadeguo. (Il Resto del Carlino)

f) Frecuencia: la mayor parte de los compuestos hallados presenta una gran volatilidad, es decir, aparecen como mucho una o dos veces en todo el corpus,

20 Atracadores en la nueva era del euro.

21 Vendedores de droga en la nueva era del euro.

22 Turistas que se empeñan en pagar con euros.

${ }^{23}$ Es un hecho bien (re)conocido que el italiano, sobre todo en los medios de comunicación, utiliza un número mayor de anglicismos que los medios de lengua española, al menos los de España.

${ }^{24}$ Es decir: el primer día en el que circuló el euro. 
por lo que pueden ser considerados 'ocasionalismos'. Solo las palabras siguientes han sido contabilizadas al menos tres veces:

- esp.: eurocalculadora, euroconversor, euromonedero, eurozona (2002); eurobanco, eurobonos, Eurogrupo, euromanía, eurosistema, eurozona (2013).

- ital.: eurocalcolatore, eurocaos, eurconvertitore, Eurolandia, europessimista, europinocchio d'oro (2002); eurobond, Eurogruppo, Eurolandia, Eurotower, eurozona (2013).

Varios de estos compuestos frecuentes, lógicamente, son comunes a las dos lenguas, ya que se trata de objetos o realidades 'estrella' de cada momento: eurocalculadora/eurocalcolatore, euroconversor/euroconvertitore (2002), eurobonos/ eurobond, Eurogrupo/Eurogruppo, eurozona (2013). Si nos fijamos, en cambio, en la evolución entre los dos periodos analizados (2002 y 2013) observamos una cuasi nula continuidad, que se limita a la palabra eurozona en español y a su equivalente periodístico italiano Eurolandia.

g) Coincidencias entre las dos lenguas: un cierto número de compuestos aparecen tanto en italiano como en español. Son los casos previsibles, al estar tomados de una fuente común (eurocent en el subcorpus de 2002; eurogrupol eurogruppo, eurosistema en ambos subcorpus; eurobonoleurobond, eurostoxx y eurozona en el de 2013) o al corresponder a circunstancias análogas del momento (todos pretenecen a los subcorpus de 2002) en ambos países: eurobilletel eurobanconota, eurocalculadora/eurocalcolatore, euroconversor o euroconvertidor/ euroconvertitore, euroforia, euromanialeuro-mania, euromonedaleuro-moneta, euromonedero/euroborsellino.

Antes de pasar al punto siguiente no podemos dejar de comentar un hecho evidente: de la explosión terminológica que se produjo en torno al 1 de enero de 2002 diez años después queda muy poco, como hemos tenido ocasión de constatar al recopilar, durante el año 2013, textos periodísticos de tema económico (y al examinar los diccionarios, como veremos luego). No consideramos inútil, sin embargo, el análisis de las palabras que se crearon en aquel lejano 2002, puesto que ambas lenguas se sometieron a un estímulo idéntico y reaccionaron de diferentes modos, en función tanto del modo de expresarse de sus periódicos como de la diferente aplicación de reglas de formación de palabras muy similares.

\section{EL PLURAL DEL NUEVO SUSTANTIVO}

En una inusual nota de tipo lingüístico redactada en 1998 por el Director General de Asuntos Económicos y Financieros de la Comisión Europea, se 
prescribía que en italiano el sustantivo euro debía ser invariable, mientras que en español su plural sería euros. ${ }^{25}$

La palabra no parece haber encontrado problemas en su adaptación al español -en línea con lo señalado por la Comisión-, pues se ha insertado con normalidad en el paradigma de los sustantivos masculinos españoles acabados en -o. Así, su plural regular fue desde un principio el mismo que los de su serie: euros (cfr. DPD, s.v.). Otra muestra de normalidad de su inserción en la morfología del español es la creación de las habituales formas alteradas, sobre todo en el habla coloquial: euritos, eurillos, euretes, euracos, etc.

Bastante menos sencilla ha sido su incorporación al italiano, ámbito lingüístico en el cual no han faltado incluso intervenciones de políticos y de lingüistas al respecto. ${ }^{26}$ En esta lengua habría podido integrarse, como en español, en la serie de sustantivos masculinos en -o. Sin embargo, la palabra tropezó con otro paradigma de sustantivos, el de los invariables en -o, fruto casi siempre de acortamientos de palabras: auto, frigo, metro, moto, radio, stereo, video, etc. El italiano, aplicando una cierta analogía, ha alineado la nueva palabra con este segundo grupo, por lo que, al igual que estas otras palabras, euro es invariable en italiano. Hay que aclarar que, en realidad, el término euro no es ningún acortamiento de otra palabra preexistente, pese a lo cual los hablantes (influidos además por otros factores) han asimilado la nueva palabra a otras que sí lo eran. También en italiano esta palabra ha dado lugar a nombres alterados: eurino, euretto, euraccio, formas estas que sí son variables en plural. Esta última característica diferencia el sustantivo euro de la mayoría de los invariables en -o, que raramente aceptan un sufijo apreciativo (Merlini Barbaresi 2004: 268).

Quizá por este carácter contradictorio no son en absoluto inusuales los ejemplos en italiano del plural 'regular' euri, ${ }^{27}$ si bien suelen aparecer en contextos

25 Así se refleja aún hoy en el Libro de estilo Interinstitucional de la Unión Europea (que puede consultarse en la web: http://publications.europa.eu/code/es/es-000100.htm [consulta: 27 de diciembre de 2013]).

26 Sobresale en particular la intervención, en el año 2001, del entonces Presidente de la Accademia della Crusca (organismo que, aunque no es equivalente en sus funciones a nuestra RAE, goza de predicamento entre los hablantes italianos), Francesco Sabatini, a favor de la invariabilidad de la palabra, o el decreto gubernamental (25 novembre 2001) del mismísimo Presidente de la República Italiana, Carlo Azeglio Ciampi, que ordenaba sustituir todas las apariciones en documentos públicos del plural euri por el plural invariable euro. No han faltado defensores del plural variable euri, con argumentos ciertamente razonables, como Yarick Gomez Gane en su libro de 2003, o Irene Scalici (en la web del Istituto Treccani), que no se muestra contraria a esta forma. Así pues, el tema ha despertado pasiones y ha hecho correr ríos de tinta en el país hermano.

27 En nuestros dos subcorpus de italiano (de italiano periodístico, no lo ovidemos) encontramos un solo ejemplo, que sin embargo resulta inválido, al tratarse de una cita paródica de unos versos de Carducci, en los que tiene el significado de 'viento austral': "Prendiamoci l'ottimismo di Carducci: «Le dolenti imagini / si portin via gli Euri in mare. / Diciam parole prospere: / Benigno amor ne appare»" (Corriere della Sera). 
marcados (bien por su coloquialidad, bien por su carácter humorístico) o en situaciones similares.

\section{EL SUSTANTIVO EURO EN LA FORMACIÓN DE PALABRAS}

El sustantivo euro, como cualquier palabra (sustantivo, adjetivo, verbo), puede constituir la base de nuevas palabras bien mediante composición nativa o bien mediante la creación de nuevas unidades léxicas pluriverbales o lexías complejas. En nuestros corpus aparecen algunos casos, ciertamente menos numerosos respecto al grupo de los compuestos con euro-, en los que vuelve a mostrarse una cierta creatividad lingüística, en particular en italiano, que aventaja de nuevo al español.

El sustantivo euro, ateniéndonos a los datos recogidos, ha dado lugar a los siguientes tipos de unidades léxicas: a) palabras prefijadas, b) palabras sufijadas, con sufijoides, c) compuestos nativos, y d) lexías complejas. Estos son los datos obtenidos: 28

\begin{tabular}{|c|c|c|c|c|}
\hline & \multicolumn{2}{|l|}{ español } & \multicolumn{2}{|c|}{ italiano } \\
\hline & 2002 & 2013 & 2002 & 2013 \\
\hline a) & proeuro, Trans-Euro & & extra euro, supereuro & anti-euro, super- \\
\hline b) & eurófilo, eurófobo & & eurardario, eurofilo & \\
\hline c) & $\begin{array}{l}\text { míster euro, alerta } \\
\text { euro, Cheque-Euro, } \\
\text { efecto euro, era euro, } \\
\text { zona euro }\end{array}$ & $\begin{array}{l}\text { área euro, } \\
\text { zona euro }\end{array}$ & $\begin{array}{l}\text { Euromio, eurovaticano, } \\
\text { area euro, baby euro, } \\
\text { effetto-euro, emergenza } \\
\text { euro, eurolira, formato } \\
\text { euro, pianoeuro, zona } \\
\text { euro, controlla-euro, porta } \\
\text { euro }\end{array}$ & $\begin{array}{l}\text { Nord-Euro, } \\
\text { Sud-Euro, area } \\
\text { euro, Paesi euro, } \\
\text { progetto-euro, } \\
\text { zona euro, salva- } \\
\text { euro }\end{array}$ \\
\hline d) & $\begin{array}{l}\text { eurosuperstar, flores- } \\
\text { euro }\end{array}$ & & $\begin{array}{l}\text { Euro Superstar, neuro, } \\
\text { sirenaeuro }\end{array}$ & \\
\hline
\end{tabular}

Hay un primer problema que debemos afrontar (y que, por ende, no es exclusivo de nuestro constituyente): determinar si algunas unidades léxicas son compuestos o lexías complejas. No nos ayuda en este caso la grafía, sobre todo en italiano, ya que compuestos aparentemente claros se escriben con sus componentes separados, mientras que posibles unidades pluriverbales se escriben con sus elementos juntos o separados por guiones. Nos hemos basado para determinar su naturaleza en el criterio de discriminación establecido por Antonietta Bisetto (2004): se tratará de lexías complejas cuando la combinación de dos sustantivos o de un sustantivo y un adjetivo cumpla estos dos criterios: a) su interpretación es no composicional; b) el núcleo no puede ser interpretado como un hiperónimo del conjunto. De esta manera, unidades léxicas como efecto euro o emergenza euro serían considerados compuestos, y quedará en el

${ }^{28}$ Como se dijo antes, recogemos aquí solo una de las formas de cada palabra; el resto de variantes se encontrarán en el vocabulario final. 
grupo de las unidades pluriverbales solo una, que aparece en las dos lenguas y que en realidad es una formación exógena en los dos ámbitos lingüísticos: Euro superstar. ${ }^{29}$ También al margen quedarían cuatro casos (que hemos englobado en este grupo) en los que se manifiesta algún tipo de juego lingüístico:

Digáselo con flores-euro, caballero. (La Razón $)^{30}$

Bienvenido Míster euro. La bienvenida a míster euro demuestra que los europeos de a pie apuestan por la unidad y el proceso de construcción de una nueva Europa. (La Razón) $)^{31}$

Nonostante tutto, Marta continua a bisticciare con la moneta europea. "Preferisco chiamarla "neuro" - si lamenta - Ognuno la spiega a modo suo. È un eurodelirio". (La Repubblica) ${ }^{32}$

C’è chi teme frodi levantine: "Non mi fido, col cambio ci fregano. Per fortuna domani la pranoterapeuta la pago con un assegno". Chi resiste alla sirenaeuro: "Fino al 28 febbraio sono obbligati a prendere le mie lire, e io insisto con quelle". (La Repubblica) $)^{33}$

Por otra parte, la palabra euro da lugar a unos pocos prefijados y sufijados (en este último caso con formantes cultos). Uno de estos sufijados (eurardario) merece un comentario a parte, puesto que esconde una formación más compleja. En efecto, el supuesto sufijo -ardario no existe en realidad en italiano. A quien era muy rico durante la época de la lira se le llamaba miliardario, a partir del término miliardo ('1000 millones'). El sufijo, por tanto, era el más común -ario. Dado que la eventual unión de este sufijo con nuestra palabra hubiera producido una forma poco impactante y casi incomprensible (*eurario), se opta por tomar un segmento que incluye parte de la base original (-ardario), por lo que en realidad se trataría de una formación acronímica (Casado Velarde 1999: 5085 ss.). ${ }^{34}$

El grupo de los compuestos nativos es el más numeroso y resulta más productivo en italiano (18 elementos frente a los 8 del español). Estas son sus características:

a) En cuanto a su categoría gramatical, todos los del español son sustantivos, al igual que casi todos los del italiano, excepción hecha de tres compuestos $\mathrm{V}+\mathrm{N}$ que son adjetivos (contralla-euro, portaeuro, salvaeuro).

${ }^{29}$ Corresponde bien a un espectáculo preparado por el BCE para dar la bienvenida al euro, bien a un concurso para escolares de la zona euro; en ambos casos su significado es, en efecto, no composicional y su núcleo (euro) no es hiperónimo del conjunto.

30 Se juega con la expresión Dígaselo con flores, que popularizó hace varios decenios la red de floristerías Interflora.

${ }^{31}$ Referencia inequívoca al célebre film de Luis García Berlanga, Bienvenido Mister Marshall.

${ }^{32}$ Cruce entre el término neuro (que puede ser tanto el prefijo culto neuro-como una abreviatura de 'clinica neurologica') y la propia palabra euro.

${ }^{33}$ En este fragmento el euro es comparado con los cantos de las sirenas, como las que pretendían atraer al barco de Ulises hacia los escollos para que naufragara; de la misma forma, parece que el euro intente atraer a sus nuevos usuarios simplemente para engañarlos.

$34 \mathrm{O}$ parola macedonia, según el feliz término acuñado por Bruno Migliorini para el italiano. 
b) El esquema de formación seguido mayoritariamente es el de la aposición $\mathrm{N}+\mathrm{N}$ (zona euro, effetto-euro, etc.), pero hay también (solo en italiano) dos ejemplos de N+A (Euromio, eurovaticano), dos de A+N (Nord-euro, Sud-euro) y los tres del ya mencionado $\mathrm{V}+\mathrm{N}$.

c) El esquema organizativo reproduce generalmente el esquema habitual [determinado + determinante] de los compuestos endocéntricos, aunque no falta el inverso en un caso, que reproduce un esquema exógeno, inglés: baby euro. Encontramos además un compuesto coordinado, es decir, con dos núcleos (eurolira) y tres ejemplos ya vistos de compuestos exocéntricos (los formados con $\mathrm{V}+\mathrm{N})$.

d) Desde el punto de vista gráfico se observan muchas vacilaciones, tanto por su rareza u ocasionalidad (uso o no de las comillas) como por la percepción del grado de fusión entre sus componentes (uso del guión y escritura separada o amalgamada de los mismos).

\section{EL EURO EN LAS FUENTES LEXICOGRÁFICAS}

Para concluir este trabajo nos proponemos verificar cómo las fuentes lexicográficas se han hecho eco de esta nueva realidad. Para ello hemos recurrido a dos tipos de fuentes: por un lado dos diccionarios monolingües representativos (DRAE para el español y Zingarelli para el italiano), en las ediciones más cercanas a los dos momentos señalados; por el otro, algunos diccionarios o recopilaciones de neologismos. A diferencia de los materiales analizados, que proceden de unos fragmentos de realidad lingüística que no han pasado por otro tamiz más allá de los propios de la publicación periodística, los diccionarios suponen una selección, una decantación -sirva la metáfora química o vinícolade lo observado en la realidad lingüística.

Paralelamente a lo dicho sobre el lenguaje periodístico, en el terreno lexicográfico descubrimos también unas diferencias casi insalvables entre las tradiciones española e italiana, que hacen muy difícil una comparación de este aspecto neológico. En efecto, mientras la lexicografía italiana (y el diccionario Zingarelli es un excelente ejemplo) recoge casi con avidez cualquier neologismo que empiece a circular en esta lengua, sin filtros demasiado exigentes, la lexicografía española (dominada por el DRAE, un diccionario académico al que no podemos pedir una actitud similar a la de los diccionarios italianos, todos ellos fruto de empresas privadas) es mucho más parsimoniosa a la hora de acoger en su seno nuevas palabras. En cuanto a los diccionarios de neologismos, hay que señalar que también Italia adelanta a España en este campo, puesto que desde los años 80 del siglo pasado no han dejado de de aparecer diccionarios de 
este tipo, a los que podemos añadir varias recopilaciones publicadas en línea; ${ }^{35}$ en España, en cambio, este tipo de obras escasean.

Así pues, y como se verá a continuación, las diferencias entre lo recogido en unos y otros diccionarios son notables, aunque hemos de considerar que no son debidas - al menos completamente- a la inexistencia de compuestos con euro en español, sino más bien y con casi total seguridad a la disimetría señalada en cuanto a la capacidad de recogida de neologismos en ambas tradiciones lexicográficas.

Comencemos por lo que aparece en las ediciones publicadas hacia al año 2002:

- Zingarelli 2000: eurobbligazione, eurobond, eurocent, eurolandia. ${ }^{36}$

- DRAE 2001 (22a ed.): no hay ninguna entrada.

Y a continuación los datos de los diccionarios actuales:

- Zingarelli 2014: eurobbligazione, eurobond, eurocent, eurolandia, eurozona.

- DRAE 2013 (avance de la 23 ${ }^{\mathrm{a}}$ ed.): no hay ninguna entrada. ${ }^{37}$

A estas últimas obras podemos añadir los datos de los dos compendios de neologismos señalados anteriormente en nota:

- Treccani: euro-accordino, euro-allarme, euro-identità, euro-portafoglio, euro-rischio, euro-shopping, eurobond, eurocontagio, eurodebole, eurodelirio, eurodeluso, eurodemolitore, eurodracma, eurodubbio, euroeclissi, eurofusione, eurogruppo, euromutuo, euronegligenza, eurosalvadanaio, eurosistema, eurostabilità, eurostangata, eurotest, eurotracker, eurovoglia, eurozona.

- NDVUA: euribor (y las variantes raras euroibor y euromibor), euroclub, euroemergencia, euromoneda, europreocupación, eurosistema, eurozona.

- Diccionario neologismos del IULA: eurocalculadora, eurozona.

Vemos, pues, que el DRAE se ha limitado hasta ahora a recoger la palabra euro, pero no el resto de compuestos y mucho menos aún las unidades pluriverbales que se han ido formando en estos años y que tan frecuentes son hoy en día, al menos en el lenguaje periodístico. Además de la reciente incorporada entrada euro, quizás esperaríamos encontrar en el diccionario académico alguna otra palabra ya bien asentada en el español peninsular, como eurozona y quizá también eurobono $0^{38}$ y eurosistema. Tampoco dos diccionarios que recogen con

35 Nos referimos por ejemplo a la extensísima lista que puede encontrarse en la página web del prestigioso Istituto Treccani (www.treccani.it), a la que podemos oponer escasos representantes en español, como por ejemplo el NDVUA o la página de neologismos del IULA de la Universitat Pompeu Fabra de Barcelona (http://obneo.iula.upf.edu/spes/).

36 El GRADIT, por su parte, recoge los siguientes neologismos: eurobag, eurobanca, euroobbligazioneleurobbligazione, eurobond, eurocent, eurocredito, eurodeposito, euroemissione, eurofilo, eurolandia, eurozona.

${ }^{37} \mathrm{La}$ única diferencia respecto a la futura $23^{\mathrm{a}}$ edición consiste en que en la nueva edición aparecerá la palabra euro en el sentido de 'moneda europea'.

38 De los que se habla desde 2010 y que por tanto no podríamos encontrar en el NDVUA, cuyo periodo de recogida de léxico acaba en el año 2000. 
cierta generosidad los neologismos del español (NDVUA y IULA) se muestran muy receptivos, en particular el segundo, pues se limita a señalar dos de ellos, siendo además el primero un objeto muy ligado al momento de la implantación del euro, por lo que hoy resulta desusado (eurocalculadora). Zingarelli, un diccionario de tamaño medio, recoge en la actualidad prácticamente las mismas palabras que ya aparecían en el año 2000 (con el único añadido de eurozona). Mucho más generosos se muestran otras dos obras: GRADIT (que es un diccionario del italiano contemporáneo de notables dimensiones, ${ }^{39}$ el más amplio publicado hasta hoy), con 11 entradas, y Treccani, con 27 palabras documentadas. ${ }^{40}$

\section{CONCLUSIONES}

La implantación del euro en Italia y en España, un hecho en principio extralingüístico, ha tenido una serie de consecuencias relevantes en las lenguas italiana y en española, que han afectado fundamentalmente al nivel léxico (aunque también las ha habido en el nivel puramente morfológico: recuérdense las dificultades en italiano para fijar el plural de la palabra euro), puesto que ha sido necesario crear no solo una palabra para la nueva moneda, sino también una serie de unidades léxicas derivadas de ella. En la mayor parte de los casos se ha tratado de palabras compuestas con el prefijoide euro-, aunque no han faltado otro tipo de palabras e incluso de lexías complejas.

El examen minucioso de los materiales obtenidos de un corpus de textos paralelos de prensa escrita en italiano y español ha puesto de manifiesto importantes diferencias entre ambas lenguas. Estas divergencias se explican de dos modos. En primer lugar, por el hecho de que el lenguaje periodístico español es mucho más sobrio que el italiano y por tanto tiende en menor medida a la creatividad e incluso al juego lingüístico, aunque no estén del todo ausentes, como hemos visto. Pero también hemos constatado, en segundo lugar, discrepancias más profundas, reflejo de una diferente aplicación de los mecanismos de formación de palabras, en particular de los compuestos con el prefijoide euro-, terreno en el que el italiano se muestra mucho más productivo que el español, no solo porque el número de palabras encontradas en el corpus es mucho mayor, sino porque sus características -que hemos expuesto en las

39 Seis gruesos volúmenes y unas 250000 entradas.

40 En varios casos el contexto no permite afirmar con total seguridad que el prefijoide eurose refiera realmente a la moneda única y no a Europa y sus instituciones. Por ejemplo, son casos dudosos: euro-accordino, euro-portafoglio, euro-rischio, eurodelirio, eurodeluso, eurodemolitore, eurodubbio, euronegligenza, eurostabilità, eurotest, eurotracker. Mientras GRADIT sigue criterios de selección lexicográficos (por ejemplo, cierta frecuencia de uso), Treccani no tiene en cuenta esos límites, ya que incluye cualquier compuesto documentado incluso una sola vez; de ahí la presencia de muchas palabras que son sin duda ocasionalismos. Un criterio similar es el que sigue el NDVUA. 
páginas anteriores- son mucho más ricas y variadas que en español. Así pues, se perfila una tendencia del italiano a un mayor uso -tanto desde el punto de vista cuantitativo como cualitativo- de este mecanismo de formación de palabras respecto al español.

\section{BIBLIOGRAFÍA}

Bisetto, A., "Composizione”, en M. Grossmann - F. Rainer (2004: 52-95).

Carrera Díaz, M., "La construcción del texto periodístico en italiano y español", en: $\mathrm{M}^{\mathrm{a}}$ de las N. Muñiz - F. Amella (eds.), La costruzione del testo in italiano: sistemi costruttivi e testi costruiti, Florencia, F. Cesati, 1996, pp. 113-126.

DPD = Real Academia Española, Diccionario panhispánico de dudas, Madrid, EspasaCalpe, 2005.

DRAE = Real Academia Española, Diccionario de la lengua española, Madrid, Espasa-Calpe, 2001 (22a ed.). También en red (con las enmiendas para la nueva edición incorporadas: www.rae.es).

Enc. It. = Simone, R. (dir.), Enciclopedia dell'italiano, Roma, Istituto della Enciclopedia Italiana, 2010.

García Palacios, J., "La quimera del euro-. Acerca de algunos elementos compositivos en español", Revista de Lexicografía 7 (2000-2001), pp. 33-62.

Gomez Gane, Y., Euro. Storia di un neologismo, Economia \& Politica economica, 2003.

GRADIT = DE MAUro, T. (dir.), Grande dizionario italiano dell'uso, Turín, UTET, 1999-2000.

Grossmann, M. - Rainer, F. (eds.), La formazione delle parole in italiano, Tubinga, Niemeyer, 2004.

Hartmann, R. R. K., Contrastive textology: comparative discourse analysis in applied linguistics, Heidelberg, Julius Groos, 1980.

IAcobini, C. - Thornton, A.M., "Tendenze nella formazione delle parole nell'italiano del ventesimo secolo", en B. Moretti et al. (eds.), Linee di tendenza dell'italiano contemporaneo. Atti del XXV Congresso internazionale di studi della Società di linguistica italiana (Lugano, 19-21 settembre 1991), Roma, Bulzoni, 1992, pp. 25-55.

Iacobini, C., "Prefissazione", en M. Grossmann - F. Rainer (2004: 97-163).

IULA = Diccionario de neologismos on line. Institut Universitari de Llengües Aplicades. 30 de diciembre de 2013. <http://obneo.iula.upf.edu/spes>

MaPelli, G., "Análisis contrastivo de la estructura del editorial en italiano y en español", en L. Blini - M. V. Calvi - A. Cancellier (eds.), Linguistica contrastiva tra italiano e lingue iberiche. Atti del XXIII Convegno dell'Associazione Ispanisti Italiani (Palermo 6-8 ottobre 2005), 2007, pp. 343-360.

Merlini Barbaresi, L., "Alterazione”, en M. Grossmann-F. Rainer (2004: 264-292).

NDVUA: Alvar EzQuerra, M. (dir.), Nuevo diccionario de voces del uso actual, Madrid, Arco/Libros, 2004.

NGRAE = Real Academia Española, Nueva Gramática de la Lengua Española, Madrid, Espasa-Calpe, 2010. 
Rati, M.S., "La formazione delle parole nell'italiano contemporaneo: sondaggi sui composti nei dizionari dell'uso", en S. Ferreri (ed.), Lessico e lessicologia. Atti del XLIV congresso internazionale di studi della Società di Linguistica Italiana (Viterbo 27-29 settembre 2010), Roma, Bulzoni, 2012, pp. 121-129.

Sabatini, F., "Gli euro e le lingue”, La Crusca per voi 23 (2001), pp. 1-2.

Tonani, E., “Trattino”, en: Enc. It., s.v.

Varela, S. - Martín García, J., “La prefijación”, en I. Bosque - V.Demonte, Gramática descriptiva de la lengua española, Madrid, Espasa-Calpe, 1999, vol. III, pp. 4993-5039.

ZING 2000 = Vocabolario della lingua italiana, Bolonia, Zanichelli, 1999 (12a ed.).

ZING 2014 = Vocabolario della lingua italiana, Bolonia, Zanichelli, 2013 (12a ed.).

\section{APÉNDICE ${ }^{41}$}

Ejemplos extraídos del corpus

\section{ESPAÑOL}

“alerta euro" $f(2002)$ v. ej. || En el dispositivo «Alerta euro» participan casi un centenar de personas, que han atendido llamadas de usuarios que mostraban dudas sobre las devoluciones en euros y pesetas (ABC).

área euro $f(2013)$ eurozona.

Cheque-Euro $m$ (2002) cheque con el importe expresado en euros || Un francés pagó incluso con Cheque-Euro (EM).

efecto euro $m$ (2002) efecto producido por la implantación del euro || Hasta el DJ sufre el efecto euro (EM).

era euro [era euro] $f(2002)$ época marcada por la implantación del euro || primera huelga de la era euro en el Hexágono (LV).

euroabuso $m$ (2002) abuso por la implantación del euro || Euroabusos. Casi todos los días laborables tomo un taxi a las 15.00 h, en Aragó/Casanova hasta València/paseo de Gràcia. Antes del euro, me costaba siempre 300 pesetas, la tarifa mínima. (LV).

euroasesor ["euroasesores"] $m$ (2002) v. ej. || 250 «euroasesores» de la cadena Eroski han informado a 350.000 consumidores sobre la nueva moneda (ABC).

eurobanco [eurobanco, Eurobanco] $m(2002,2013)$ Banco Central Europeo.

eurobillete $m$ (2002) billete de euro || El primer eurobillete que se concedió en una de las más de ocho mil cajas bancarias de Portugal correspondió a una sucursal de Oporto, quince segundos después de las campanadas (ABC).

41 Los artículos de este repertorio siguen el siguiente esquema: lema [variantes formales] categoría gramatical (año del corpus) definición || ejemplo. Tras los ejemplos se indica la fuente, según estas siglas: EM= El Mundo; EP= E1 País; RAZ= La Razón; LP= Las Provincias; LEV= Levante-EMV; LV= La Vanguardia; CS= Corriere della Sera; ESP= L'Espresso; IS= I1 Sole 24 ore; $\mathrm{SIC}=\mathrm{La}$ Sicilia; $\mathrm{IM}=\mathrm{Il}$ Messaggero; $\mathrm{PAN}=$ Panorama; $\mathrm{REP}=\mathrm{La}$ Reppublica; $\mathrm{RC}=\mathrm{Il}$ Resto del Carlino. La forma del lema corresponde a la más habitual en el corpus. Si un ejemplo ha sido ya citado y explicado en una nota anterior, se indica mediante la remisión "v. nota x". Si el ejemplo contiene la explicación del significado, se omite la definición, sustituida por la indicación "v. ej.". Para las palabras ya bien asentadas no se ofrece ningún ejemplo. 
eurobono $m$ (2013) bonos emitidos por la zona euro || Pero eso es solo una pequeña parte de lo que demanda Hollande, que pide eurobonos, aunque no para mutualizar la deuda -algo frontalmente rechazado por Alemania- sino para financiar infraestructuras. (LP).

eurocalculadora $f(2002)$ v. ej. || Hasta ahora, admiten, no han tenido ningún problema. Se ayudan con una eurocalculadora ajustada al cambio que a ellos les interesa (EP).

eurocampanadas $f p l(2002)$ v. nota 15.

eurocandidato $m$ (2002) candidato a ingresar en la zona euro || Frustración y esperanza en los eurocandidatos $(\mathrm{LV})$.

eurocent ["eurocent"] $m$ (2002) céntimo de euro

eurocepillo ["eurocepillo"] $m$ (2002) cepillo de iglesia en el que ahora se introducen euros || La normalidad se extiende. El "eurocepillo" de la lglesia (EM).

eurocínico $m$ (2002) quien mantiene una postura cínica respecto al euro || Porque si son preocupantes los análisis de los euroescépticos, mucho más alarmantes son las odas triunfales de los eurocínicos que conocen el precio de todo y el valor de nada. (EM).

eurocinismo $m$ (2002) cinismo respecto al euro $\| \mathrm{El}$ eurocinismo. Lo peor que le podría ocurrir a la nueva moneda europea, que ha entrado en vigor con el nuevo año, ha ocurrido ya con la inflación de eurocínicos que acompaña al euro (EM).

eurocirculatorio adj (2002) que guarda relación con la circulación del euro || sólo en el primer día eurocirculatorio me metieron 2.000 pesetas largas de clavo entre la gasolinera (400), la farmacia (otras tantas), el café y las porras mañanero (casi 300) (RAZ).

euroconsultorio $m$ (2002) sección del diario con respuestas a las consultas sobre la nueva moneda.

euroconversor $m$ (2002) eurocalculadora || Únicamente una señora que creía que le devolvíamos mal, pero con la ayuda de los euroconversores le pudimos explicar que no era así (RAZ).

euroconvertidor $m$ (2002) eurocalculadora || Pues esperen un momento que desenfunde el euroconvertidor mágico (LP).

eurodelincuencia $f(2002)$ v. ej. || Eurodelincuencia. La nueva moneda ya llega a todos. Los atracadores ya han protagonizado esta semana varios robos en euros, aunque parecen seguir teniendo inclinación por las vieja pesetas. (LP).

eurodesencanto $m$ (2013) desencanto por la nueva moneda || Las secuelas en las relaciones entre economía y democracia son uno de los motores del eurodesencanto (EP).

"euroejemplo" $m$ (2013) ejemplo para toda la zona euro || Pero considerar a Alemania como el "euroejemplo" es una grave tergiversación de los acontecimientos (EP).

euroempleo ["euroempleos"] $m$ (2002) empleo vinculado al uso y difusión de la nueva moneda || Estos "euroempleos" se circunscribirán a trabajos de información y comprobación (LV).

euroeuforia [euro-euforia] $f(2002)$ euforia motivada por la llegada del euro || Euroeuforia. España ha asumido su tercera presidencia semestral del Consejo de la UE coincidiendo con un paso histórico en la construcción europea (EP). 
eurofiesta $f(2002)$ v. ej. || Eurofiesta en la Puerta del Sol. Unas 30.000 personas dieron la bienvenida al Año Nuevo y a la moneda única en la madrileña Puerta del Sol (EM).

eurófilo [eurófilo] adj (2002) favorable al euro.

eurófobo [eurófobo] adj (2002) contrario al euro.

euroforia $f(2002)$ euforia motivada por la llegada del euro || La euroforia de estos días no puede borrar la reciente imagen de Schröder, Blair y Jospin negociando el grado de subordinación de Europa a Washington al margen de los restantes líderes europeos (EM).

euroganador ["euroganadores"] adj/s (2013) v. nota 17.

Eurogroup $50 \mathrm{~m}$ (2002) grupo de expertos de la zona euro || El pasado 30 de noviembre, los miembros del Eurogroup 50, que nos reunimos dos veces al año para analizar y debatir todos los asuntos de interés para la Zona Euro y su moneda única (EP).

Eurogrupo [Eurogrupo, eurogrupo] $m(2002,2013)$ conjunto de países que han adoptado el euro como moneda.

eurohistérico adj/m (2002) que manifiesta histeria a causa de la llegada euro || La cola de eurohistéricos llega a la Calle de San Jerónimo, pero la eficacia es extraordinaria (EM).

euroinundación $f$ (2002) llegada masiva de la nueva moneda || Ha sido una semana semifestiva y lluviosa en la que el mercado, día por día, ha ido viviendo, a pesar de todos los contratiempos, el gran acontecimiento de la euroinundación (LP).

euromanía [euromanía, Euromanía] $f$ 1. (2002) expectación creada por la llegada de la nueva moneda || Euromanía en Euskadi. La puesta en circulación del euro atrajo a miles de ciudadanos vascos a las cerca de 40 sucursales bancarias que abrieron ayer para facilitar el canje de pesetas en efectivo por la nueva moneda europea (EP). 2. (2013) v. ej. || 100 Montaditos introdujo en 2008 el concepto «Euromanía», que se seguirá manteniendo miércoles y domingo, y en el que todos los productos cuestan 1 euro (ABC).

euromercado ["euromercado"] $m$ (2002) mercado o tráfico económico en el que ahora se paga en euros || El "euromercado" de la droga. Los traficantes del viejo cauce del río discutían ayer sobre los nuevos precios para las dosis (LEV).

euromoneda [euromoneda, "euromoneda"] $f(2002)$ euro || Algunos de los traficantes discutían ayer airadamente sobre los nuevos precios de las dosis con la euromoneda (EM).

euromonedero [euromonedero, "euromonedero"] $m$ (2002) conjunto de monedas de la nueva divisa preparado para su puesta en circulación || se calcula que fueron distribuidos ayer unos 250 millones de euros, que se suman a los 19 millones que fueron previamente distribuidos en forma de "euromonederos" (RAZ).

euromundo $m$ (2002) mundo en el que existe la nueva moneda || Bienvenido al 'euromundo' (EM).

europroducto ["europroductos"] $m(2002)$ v.ej. || El Corte Inglés vende «europroductos», como calculadoras, bolígrafos detectores de billetes falsos, cajas registradoras (ABC). 
europtimista $m$ (2002) optimista ante la llegada del euro \| Por un momento creí que habíamos encontrado al europtimista número uno (RAZ).

Eurosistema [Eurosistema, eurosistema, "eurosistema"] $m(2002,2013)$ v. ej. || E1 BCE informó hoy de que el Eurosistema, formado por el BCE y los bancos centrales nacionales de los países de la zona del euro, ha concluido los preparativos para la introducción de un nuevo billete de 5 euros $(\mathrm{ABC})$.

Eurostoxx $m$ (2013) índice de referencia bursátil de la zona euro || es posible pensar en volver a ver el Eurostoxx aproximarse a los 3.000 puntos antes de fin de año (RAZ).

Eurostoxx 50 [Eurostoxx 50, Euro Stoxx 50] $m$ (2013) índice de referencia burátil de la zona euro || Existe una correlación directa entre el euro y el Eurostoxx 50, como podemos comprobar en el gráfico adjunto (LP).

eurosuperstar ["eurosuperstar"] $m$ 1. (2002) v. ej. $\|$ Uno de los actos oficiales, al más puro estilo estadounidense, lo protagonizó el propio $\mathrm{BCE}$, presentando al «eurosuperstar», un acto que estuvo presidido por un eslogan de lo más británico, pese a que este país no utilizará los euros: «The euro. Our money» (ABC). 2.v. ej. || En el dispositivo «eurosuperstar euro» participan casi un centenar de personas, que han atendido llamadas de usuarios que mostraban dudas sobre las devoluciones en euros y pesetas $(\mathrm{ABC})$.

Eurotower $f(2002)$ v. ej. || La Eurotower, donde tiene su sede el Banco Central Europeo (BCE), en la Kaiserstrasse 29, en pleno centro financiero de Francfort, pasa bastante inadvertida en el "skyline" de la ciudad. (LV).

eurozona [eurozona, Eurozona, "eurozona"] $f(2002,2013)$ conjunto de países que han adoptado el euro como moneda.

flores-euro [flores-euro] $f p l$ (2002) v. nota 30.

míster euro [míster euro, Míster euro] $m$ (2002) v. nota 31.

proeuro [proeuro] adj (2002) favorable al euro || Toda la campaña proeuro nos ha animado (EM).

Trans-Euro adj (2002) servicios de vigilancia y control del transporte de euros efectuados por la Guardia Civil || Además de la famosa cifra equivalente a un euro de 166,387 pesetas, la operación Trans-Euro, que se inició el 1 de septiembre de 2001 y finalizará el 30 de junio de 2002, también se puede resumir en números (LEV).

zona euro [zona euro, Zona Euro, 'zona euro'] $f(2002,2013)$ eurozona.

\section{ITALIANO}

anti-euro adj inv (2013) contrario al euro.

area euro $f(2002,2013)$ eurozona.

baby euro $m$ inv (2002) v. ej. || Baby euro, finisce la lunga gestazione (ESP).

controlla-euro adj inv (2002) que sirve para controlar al euro \| Proprio per difendersi da questi pericoli è stato appena messo a punto un nuovo strumento controlla-euro. (PAN). 
effetto-euro $m$ (2002) efecto producido por la implantación del euro $\|$ Anche in questo caso, al di là dell' effetto-euro, saranno le valutazioni sull'andamento dell'economia, oltre che dei tassi, ad orientare il mercato (RC).

emergenza euro [emergenza euro, emergenzaeuro] $f(2002)$ situación de emergencia provocada por la implantación del euro || I cassieri, comunque, erano pronti all'eventuale emergenzaeuro: (REP).

eurardario $m$ (2002) millonario en euros $\|$ «Chi vuol essere eurardario» riproduce online il popolare quiz a premi condotto da Jerry Scotti ma con cifre delle vincite già espresse in euro (PAN).

eurina $f(2002)$ v. nota 13.

euroacquisto [euro-acquisti] $m$ (2002) compra realizada con euros $\|$ La moneta unica supera il test del primo week end di euro-acquisti. (RC).

euroadeguarsi [mi euroadeguo] v (2002) adaptarse a la situación creada con la llegada del euro || Comunque, se il presidente Ciampi dice che l'euro va bene [...] anche noi ci inchiniamo. Ma la gioia, no. Al massimo, parafrasando una famosa battuta, possiamo dire: non capisco, ma mi euroadeguo" (RC, 2002)..

"euroansia" $f(2002)$ ansia provocada por la llegada del euro \| Caos ai caselli d'autostrada. C'è chi ha parlato di "euroansia" e registrato "euronervosismi", con qualche "euroemicrania". Si è letto di un "europanico", addirittura di "eurodelirio" (CS).

euro-area $f(2013)$ eurozona

euroarrotondamento [euroarrotondamenti, euro-arrotondamenti] $m$ (2002) redondeo en los precios en euros || E questo è proprio uno dei perni attorno ai quali ruoteranno le prime cause contro gli euroarrotondamenti che saranno portate davanti al giudice di pace (REP).

euro-austerità $f$ inv (2013) austeridad en la zona euro || Il guaio globale è l'euroausterità (IS).

eurobanconota [eurobanconota, euro-banconote] $f(2002)$ billete en euros.

eurobattesimo $m$ (2002) inicio del uso del euro || Nel giorno dell'eurobattesimo della Capitale, di nuovo affollata di turisti stranieri e di famiglie con il kit di monetine regalate a Natale, i veri eroi del passaggio alla moneta unica ieri sono stati cassiere e camerieri (REP).

eurobiglietto $m$ (2002) billete en euros || l'impatto del changeover ha fatto scendere il quantitativo delle banconote circolanti da 3,5 miliardi a 3,23,1 miliardi, a cui comincia a sovrapporsi la percentuale di eurobiglietti (REP).

eurobollettino [euro-bollettini] $m$ (2002) impreso postal con las cantidades expresadas en euros || E se quella di munirsi di euro-bollettini è stata una delle indicazioni di Poste Italiane più disattese dal pubblico, meglio è andata con la richiesta di avere le somme delle pensioni in determinati tagli di banconote (RC).

eurobond [eurobond, Eurobond (pl eurobonds), euro bond] m inv (2013) bono emitido por la zona euro.

euroborsellino $m$ (2002) conjunto de monedas de la nueva divisa preparado para su puesta en circulación || Ma sono incovenienti del primo giorno: ora con l'euroborsellino e l'euroconvertitore (convertitevi, pentitevi!) tutto diventerà più facile (IM). 
eurobrindisi $m$ inv (2002) fig. brindis hecho por el euro || Al di là degli immancabili eurobrindisi, non dobbiamo quindi sottovalutare le argomentazioni di un euroscettico come Antonio Martino (RC).

euro-business $m$ inv (2002) buen negocio hecho aprovechano la llegada del euro \| Leuro-choc diventa euro-business in corso Buenos Aires: vanno a ruba borsellini ed euroconvertitori (CS).

eurocaffè $m$ inv (2002) café pagado con la nueva moneda $\|$ Per bere un eurocaffè al Vitti, bisogna aspettare che la cassiera consulti il principale sul resto (CS).

eurocalcolatore $m$ (2002) eurocalculadora.

eurocaos $m$ inv (2002) caos provocado por la llegada del euro || L'obbligo di dare il resto nella nuova moneta ha spinto molti automobilisti a rifornirsi presso i caselli "trasformati" in sportelli bancari e postali. Controesodo, eurocaos in autostrada (IM).

Euro-capodanno $m$ inv (2002) primer día del año 2002 || È stato un Euro-capodanno all'insegna dei primati (CS).

eurocassiera [euro cassiere] $f(2002)$ v. nota 18 .

eurocelebrazione $f$ (2002) celebración con motivo de la llegada del euro || Nelle eurocelebrazioni di questi giorni c'è stata una nota stonata $(\mathrm{RC})$.

eurocent $\operatorname{minv}$ (2002) céntimo de euro $\|$ I pezzi più piccoli, da 1 , 2 e 5 eurocent, sono in rame color rosso (PAN).

euro-choc $m$ inv (2002) choc producido por la llegada del euro || Leuro-choc diventa euro-business in corso Buenos Aires: vanno a ruba borsellini ed euroconvertitori." (CS).

euro-cittadino $m$ (2002) ciudadano usuario de la nueva moneda || I segreti dell'euro in trenta domande. Rispondi, calcola il punteggio e scopri che tipo di euro-cittadino sei." ( $\mathrm{RC})$.

"eurocompatibile" adj (2002) (máquina o similar) compatible con el euro || Secondo la più recente rilevazione della Commissione europea, si considera "totalmente eurocompatibile" il 43 per cento delle medie aziende europee (ESP).

euroconfusione $f(2002)$ desbarajuste provocado por la llegada del euro || Insomma il casello può diventare il confessionale dell'euroconfuso e la coda farsi prolungatissima, a momenti da casello a casello...; può diventare anche il confessionale dell'euroconfusione (IM).

euroconfuso adj (2002) en situación de euroconfusión || v. euroconfusione (IM).

euroconoscitore $m$ (2002) buen conocedor de la nueva moneda || Vi si può navigare come europrincipiante, euroconoscitore o eurointenditore: test, questionari e simulazioni di acquisti in euro, quindi, sono personalizzati con diversi gradi secondo il livello di preparazione di chi lo fa (PAN).

euro-consigliere $m$ (2002) asesor en la implantación de la nueva moneda || Gran lavoro un po' ovunque, per gli “euro-consiglieri”, gli ausiliari piazzati tra le casse o in punti strategici dei magazzini dalle grandi catene (IM).

euroconsumatore [euroconsumatore, "euroconsumatore"] $m$ (2002) consumidor con euros || Ieri mattina [...] il primo ministro ha dato il buon esempio di euroconsumatore saldando due mazzi di rose con 76 euro e spiccioli (CS). 
eurocontanti $m p l$ (2002) dinero contante en euros || Impossibile invece anche per i più coraggiosi pagare il biglietto del treno con eurocontanti alle macchinette automatiche (REP).

euroconversione $f(2002)$ conversión de liras en euros || I1 ricorso alla doppia indicazione dei prezzi [...] si manterrà probabilmente fino al ritiro delle lire il 28 febbraio 2002 per facilitare i cittadini all'euroconversione (PAN).

euroconvertitore [euroconvertitore, euro-convertitore] $m$ (2002) eurocalculadora || Pilade Riello ed Ennio Brion [...] hanno messo alla prova l'euroconvertitore spedito nelle case degli italiani dal presidente Berlusconi (REP).

eurocrisi [eurocrisi, Euro-crisi] $f$ inv $(2002,2013)$ 1. (2002) crisis provocada por la implantación del euro || Euro-crisi nel governo" (SIC). 2. (2013) crisis en la zona euro || per com'è stata strutturata la risposta all'eurocrisi, i contribuenti tedeschi oggi stanno ricevendo un sussidio silenzioso da parte di quelli italiani (REP).

“eurocuriosità" $f p l$ (2002) curiosidades sobre el euro || Carla De Girolamo, giornalista di "Panorama", naviga in Internet alla ricerca di "eurocuriosità" e informazioni utili (ESP).

euro-day $m$ inv (2002) día dedicado al euro || Lo rileva un sondaggio effettuato da Cirm per la Confcommercio e presentato nel corso dell'Euro-day organizzato dai commercianti per fare il punto della situazione (PAN).

eurodelirio [eurodelirio, "eurodelirio"] $m(2002,2013)$ situación delirante en relación con el euro || v. euroansia.

“euroemicrania" $f(2002)$ dolor de cabeza causado por el euro || v. euroansia.

euroentusiasta [euroentusiasta, euro-entusiasti, “euroentusiasti”] adj/s (2002) entusiasta por la llegada del euro || Su questo, a parte gli emigrati all'estero, euroentusiasti, sembrano tutti d'accordo (CS).

euroesordio [euro-esordio, "euro-esordio"] $m$ (2002) estreno del euro || L'euro-esordio del capo dello Stato: due caffè al costo di 154 centesimi (CS).

eurofanatico $m$ (2002) fanático del euro || Ma molti degli eurofanatici, che hanno cercato di prelevare le nuove banconote subito dopo la mezzanotte, sono stati costretti ad una vera e propria caccia al Bancomat in euro. (REP).

euro-festa $f$ (2002) fiesta con motivo de la llegada de la nueva moneda || Euro-festa con gaffe. Germania, va in tilt in diretta tivù il Bancomat della Banca Centrale (IM). eurofilo ["eurofilo"] adj (2002) favorable al euro.

euroforia [euroforia, "euroforia"] $f(2002)$ euforia motivada por la llegada del euro || A Madrid, alla Puerta del Sol, è andato in scena "Euroforia": la Spagna ha dato addio alla peseta nel giorno in cui assume, per i prossimi sei mesi, la presidenza dell’Ue (SIC).

euroforico $m$ (2002) euforico por la llegada del euro || Gli euroforici e i bancomatti. (CS). eurofratellanza $f(2002)$ hermandad basada en la moneda común || Il sentimento di eurofratellanza si affievolisce prematuramente venti minuti dopo, quando il turista scende dal lato sinistro dell'auto, rischiando portiera e mano (CS).

euroGalateo $m$ (2002) buenas prácticas en la implantación y uso del euro II L'euroGALATEO. Paura, sgomento, confusione. Non sono permessi all'eurogentleman che dovrebbe essere già ben fornito delle nuove banconote (ESP). 
euro-gatto $m$ (2002) v. nota 19.

eurogentleman $m$ inv (2002) gentleman que usa la nueva moneda || v. euroGalateo.

Eurogruppo $m(2002,2013)$ conjunto de países que ha adoptado el euro como moneda. euro-guida $f$ (2002) guía al euro|| Anche il Parlamento europeo offre una sezione euro interattiva con i siti dei 12 partner [...] e un'utile euro-guida per la stampa (PAN).

euro-identità finv (2013) identidad de los países de la zona euro || Nascita di un'euroidentità (IS).

eurointenditore $m$ (2002) experto en la nueva moneda || v. euroconoscitore.

"EuroItalia" $f(2002)$ nombre de una publicación d el semanario L'Espresso sobre el euro.

euro-kit $m$ inv (2002) conjunto de herramientas necesarias para vivir con el euro || L'ufficio postale ha venduto tutti i 100 euro-kit da 25 mila lire (CS).

Eurolandia [Eurolandia] $f(2002,2013)$ en el lenguaje periodístico, zona euro || Più della metà della popolazione di Eurolandia è preoccupata di "essere oggetto di raggiri” al momento del passaggio alle banconote e monete in euro (SIC).

eurolira $m$ (2002) presencia simultánea de ambas monedas || Nelle file, si ascoltano chiacchiere sul tema eurolira (REP).

"Eurologo" $m$ (2002) marca que distingue las tiendas que se comprometen a no encarecer sus productos al pasar al euro || le associazioni di commercio hanno siglato il 21 dicembre scorso alleanze "anti-rincari" da rispettare nei due mesi di doppia circolazione lira-euro: il fiore all'occhiello è l'iniziativa "Eurologo" che impegna l'esercizio alla trasparenza dei prezzi." (SIC).

Euromagazine $m$ inv (2002) nombre de una sección sobre el euro en el semanario L'Espresso.

'Euro-mania' $f$ (2002) euforia desmedida por la llegada del euro || Nuova era. 'Euromania' alla prova di Poste, banche e negozi (RC).

Euromio $m$ (2002) nombre de una sección sobre el euro en el semanario L'Espresso.

euro-moneta [euro-moneta, euro moneta] $f(2002)$ el euro || 9) Qual è l'euro moneta più piccola. 10) Qual è l'euro-moneta più grande. (RC).

euromonetina $f(2002)$ moneda pequeña de euro || Si è già procurata tutto il necessario: minikit per i primi acquisti, borsellino per le euromonetine (CS).

euro-nausea $f(2002)$ v. ej. || Ma i gestori dei bar si lamentano: "Circola poco contante nella nuova valuta e le code in banca restano lunghe. Questa è euro-nausea” (CS).

“euronervosismo" $m$ (2002) nerviosismo relacionado con la implantación del euro || v. euroansia.

euronews ["euronews", euroNEWS] f inv (2002) nombre de una sección sobre el euro en el semanario Panorama.

euro-offerta $f p l$ (2002) ofrenda en euros hecha en la misa || Le euro-offerte alla messa della domenica (ESP).

euro-onesto adj/s (2002) persona que se comportan correctamente en relación con el euro || Euro-onesti Coppia restituisce 6 milioni ricevuti per errore (IM). 
euro-ottimismo $m$ (2002) optimismo sobre el futuro de la economía del euro || Credo però che l'euro-ottimismo sia diventato euro-scetticismo. Io sono un euro-pessimista convinto, faccio una gran fatica a stare al passo con le innovazioni (CS).

europanico $m$ (2002) pánico producido por la implantación del euro \| v. euroansia. euro-pensione $f(2002)$ pensión pagada en euros || Le Poste: "Euro-pensioni ok". Ma a Roma e Napoli interviene la polizia (RC).

euro-pessimista $m / f(2002)$ pesimista sobre el futuro de la economía del euro || v. euro-ottimismo.

europinocchio d'oro ["europinocchio d'oro", Europinocchio d'oro, Euro-Pinocchio d'oro] $m$ (2002) v. nota 19.

euro-prezzo $m$ (2002) precio expresado en euros || la tentazione di esporre per primi il cartellino con l'euro-prezzo scontato è forte (IM).

europrincipiante adj/s (2002) persona que tiene escaso conocimiento del euro \| v. euroconoscitore.

europroblema $f(2002)$ problema relacionado con el euro o con su implantación || le domande dei lettori sugli europroblemi di ogni giorno (IM).

europrocedure $f p l$ (2002) procedimientos a seguir en la implantación del euro || Un "manuale delle europrocedure" è stato redatto dall'Aimpes (Associazione italiana manifatturieri pelli e succedanei) (RC).

euroquiz $m$ inv (2002) cuestionario con el que se comprueban los conocimientos sobre euro || Impazza l'euroquiz. Non c'è trasmissione di informazione televisiva che non lo proponga (ESP).

euro-rapinatore $m$ (2002) v. nota 20.

euroresti $m p l$ (2002) vueltas en euros de las compras || Tre i fronti infuocati: negozi, poste e banche. Ai primi spetta il compito di riempire di euroresti le tasche dei consumatori (REP).

eurosaldi [eurosaldi, 'eurosaldi'] $m p l$ (2002) rebajas con precios en euros $\|$ E i tanto attesi eurosaldi sono finalmente al via (IM).

euroscetticismo [euroscetticismo, euro-scetticismo] $m$ (2002) excepticismo en relación con el euro || L'euro? Un'invenzione di Harry Potter, uno scherzo del destino che aprirà il nuovo anno. La nuova moneta stuzzica la fantasia, sembreremo tutti più ricchi, mentre chi si sentiva ricco perché aveva tanti soldi sotto il materasso, sembrerà più povero perché diminuirà il volume. Credo però che l'euro-ottimismo sia diventato euro-scetticismo. Io sono un euro-pessimista convinto, faccio una gran fatica a stare al passo con le innovazioni, siano esse tecnologiche o monetarie (CS).

euroscettico [euroscettico, euro-scettico] adj/s (2002) excéptico en relación con el euro II Da ieri, infatti, l'euro-scettico si trova a vivere ufficialmente ai margini, come un astemio in un pub (RC).

euroSCIOCCHEZZA $f$ (2002) estupidez dicha a propósito del euro \| L'euroSCIOCCHEZZA. A forza di dirlo e ridirlo, qualcuno finirà per crederci davvero (ESP).

eurosciopero [eurosciopero, euro-sciopero] $m$ (2002) huelga en protesta por el exceso de trabajo provocado por la implantación del euro || Oggi banche chiuse, scatta l'euro-sciopero: "Troppo lavoro" (RC). 
"eurosconto" mpl (2002) descuento sobre un producto con el precio en euros || Saldi al via - Saranno Venezia, Trieste e Potenza a testare da domani gli "eurosconti". Un rebus con quattro prezzi sul cartellino: pieno e ridotto, in euro e in lire (CS).

euroshopping [euroshopping, euro-shopping] $m$ inv (2002) compras realizadas con euros || Il sorpasso avviene proprio nel giorno più difficile per i 300 consumatori europei, il primo sabato di euroshopping alla vigilia dell'Epifania (REP).

euro-sindrome $f(2002)$ v. ej. || Euro-sindrome. Allergia al nichel (IM).

Eurosistema [Eurosistema, eurosistema] $m(2002,2013)$ organización europea que supervisa el euro || le Banche centrali dell'eurosistema (IS).

Eurosondaggio $m$ (2002) sondeo sobre el euro.

euro-sostenitore $m$ (2002) partidario del euro \| Scompaiono i valori arrotondati ad un milione [...] e arrivano i decimali che metteranno alla prova anche gli eurosostenitori $(\mathrm{RC})$.

euro-spacciatore $m$ (2002) v. nota 21 .

eurospesa $f$ (2002) compra en el supermercado hecha con euros || l'ansia per l'eurospesa mette i romani in coda alle casse (IM).

eurospicci $m p l$ (2002) moneda fraccionaria de euro || Furbi che a Napoli, magari per un caffè, hanno arrotondato (a proprio favore) il resto per una presunta mancanza di eurospicci (REP).

eurospiccioli $\mathrm{mpl}$ (2002) moneda fraccionaria de euro || molti automobilisti hanno scelto infatti di pagare in contanti proprio per procurarsi eurospiccioli (REP).

euro stoxx $m$ inv (2013) índice de referencia bursátil de la zona euro.

euroStress $m$ inv (2002) estrés provocado por los cálculos de conversión en euros || euroSTRESS. Conversioni e contorsioni (ESP).

euro-sud $m$ (2013) países del sur de la zona euro || Con conseguenze per certi aspetti potenzialmente ancora più devastanti. Soprattutto nei Paesi più vulnerabili dell'eurosud. Italia compresa (IS).

Euro Superstar $m$ (2002) concurso organizado por el BCE entre los escolares de toda la zona euro || Botta e risposta col presidente della Bce, la Banca centrale europea, durante la conferenza stampa che conclude la premiazione dell'"Euro superstar", con Duisenberg che appare come una specie di Babbo Natale attorniato dai bambini vincitori (REP).

"eurotica” adj (2002) juego de palabras con 'erotica' || Al supermercato una giovane ha detto però di trovare la nuova moneta sensuale, "eurotica". Una bella invenzione (CS).

"eurotilt" $m$ inv (2002) crisis provocada por el euro || Uffici postali in "eurotilt" (SIC).

eurotismo $m$ (2002) juego de palabras con 'erotismo' || Ma oggi, e almeno per 6 mesi, ci sarà questo eurotismo e il dio Eurìalo, quello delle elargizioni, si agiterà nella tomba, e quando ognuno di noi avrà finito l'analisi delle eurine, cioè dei propri valori economici... useremo le carte di credito (CS).

euro-tombola $f(2002)$ tómbola jugada con euros || In programma figurano infatti 400 feste locali con giochi a base di euro, come l'euro-tombola e un nuovo gioco delloca (SIC).

Eurotower $m$ inv (2013) edificio que alberga a los gobernantes del euro. 
eurotransazione $f(2002)$ transacción realizada en euros || La prima eurotransazione della storia è consegnata a fotografi e telecamere (CS).

euroturista ["euroturisti"] $m$ (2002) v. nota 22.

eurounghie $f p l$ (2002) juego de palabras: v. ej. || E affilano le eurounghie per l'estate (REP).

eurovaticano $m$ (2002) euro acuñado por Ciudad del Vaticano || come sarà appunto l'eurovaticano, ci fosse il volto di un capo religioso qual è il pontefice (REP).

eurovittoria $f(2002)$ premio en euros de la lotería || La Befana regala a una tabaccheria di Ravenna la prima eurovittoria del Superenalotto (REP).

eurovolontario adj/s (2002) palabra de significado dudoso; v. ej. || "Eurofilo", "euroscettico"? Non mi riconosco in questi termini. Se proprio necessario, preferirei il termine "eurovolontario". Quello che conta prima di tutto è ciò che gli europei vogliono realmente fare insieme per sostenere la crescita, lottare contro la disoccupazione (CS).

euro-volpe $m$ (2002) v. nota 19.

euroZAPPING $m$ inv (2002) titular de un artículo de opinión de L'Espresso en el que se habla del impacto del euro en la programación televisiva.

eurozona [eurozona, Eurozona, euro-zona] $f(2002,2013)$ conjunto de países que han adoptado el euro como moneda.

extra euro adj inv (2002) ajeno a la zona euro || Un caso recente in Svezia (paese extra euro) (PAN).

formato euro $m$ (2002) v. ej. || sei bollettini formato euro (SIC).

neuro $m$ (2002) v. nota 32 .

Nord-Euro $m$ (2013) hipotética zona formada por los países del norte de Europa, que tendrían un tipo particular de circulación del euro || Non so se si debba cominciare con i piccoli, Grecia e Cipro, o varare subito una soluzione globale, dividersi nell'insieme. Tra una specie di Nord-Euro e una specie di Sud-Euro (REP).

Paesi euro $m p l$ (2013) conjunto de países que han adoptado el euro como moneda.

pianoeuro (2002) plan para la implantación del euro || Secondo l'Associazione, del resto, "il pianoeuro va avanti come stabilito ed entro il 6 gennaio, come previsto, il 90\% dei Bancomat distribuirà solo euro" (REP).

portaeuro [porta euro, portaeuro] adjinv (2002) monedero para llevar euros || borsellino "porta euro"(IM); due differenti modelli del portaeuro (PAN).

progetto-euro $m$ (2013) proyecto común de los países del euro || La Bce non interviene sui mercati con l'acquisto di bond governativi per assicurare la solvibilità di paese in difficoltà, ma per «difendere la moneta unica», il progetto-euro (CS).

salvaeuro [salva-euro, salvaeuro] adjinv (2013) (política u otra cosa) dirigida a preservar la existencia del euro ante posibles amenazas || Merkel, appello al voto «salva-euro» (IS).

sirenaeuro $m$ (2002) v. nota 33 .

Sud-Euro $m$ (2013) v. Nord-euro. 
supereuro [super-euro, supereuro] $m(2002,2013)$ euro con un tipo de cambio excesivo respecto al dólar || Il supereuro sta mettendo in ginocchio tutti, a cominciare dalle industrie e dall'occupazione (IS).

zona euro $m(2002,2013)$ eurozona. 
